for example, high resolution spectroscopy. The central problem in this field is that of relating the symmetry of an overall molecular state to the symmetries of its electronic, vibrational, rotational, torsional and spin-wave functions. This end can only be achieved if one classifies all these wave functions according to the same symmetry group-namely, a group of operations which commute with the full molecular hamiltonian, not just the "Born-Oppenheimer" hamiltonian, which by itself merely determines the electronic wave function for a fixed nuclear configuration. The elements of Dr Altmann's groups (in so far as one can determine their physical interpretation from the primitive diagrams and the associated discussion) apparently involve, in general, restorative rotations of the molecule as a whole a fact which renders them virtually useless for classifying the rotational levels of non-rigid molecules. As for Dr Altmann's remark, in the preface, that the symmetry groups of molecules such as difluoroboron methyl "had to be done in the past by computer", the group character table of the much more complex molecule boron trimethyl was first derived by hand in a few days, and Dr Altmann does not mention that he

\section{Current \\ cancer research}

The Story of Cancer: On its Nature, Causes and Control. By A. C. Braun. Pp. 308. (Addison-Wesley: Reading, Massachusetts and London, 1977.) Hardback \$19.50; paperback \$9.50.

Professor Braun wants to make a coherent story out of the chaotic literature of cancer research. In his own words, he wants to review "the major pieces of information that we have available in an attempt to solve the jigsaw puzzle that we know as cancer. If all of the required pieces have now been identified we need only to fit them into their proper places to gain an understanding of the nature of the puzzle, but if major parts are still missing then we will, of course, be unsuccessful." The present book is an expanded and more detailed documentation of concepts introduced by Professor Braun in his previous two books, The Biology of Cancer (1974) and The Cancer Problem (1969), and there can be few people concerned with cancer research who would not find much of interest in the present book. It is short (276 pages, 24 typewritten lines per page) and, because it derives from four lectures delivered to a select group of doubted its correctness for nearly ten years after its publication in 1963 .

The book is, unfortunately, riddled with misprints (a particularly glaring one is in equation 6.22 of chapter 20 , which conflicts with 9.1 of the same chapter), spelling mistakes ("lose" is repeatedly misspelled as "loose") and phrases such as (p 336) "thus satisfying all vibronic interactions" which are hardly calculated to inspire confidence in Dr Altmann's understanding of molecular quantum mechanics. How (p 326) can two molecular configurations "correspond to the same values" of the angular momenta, one would like to know?

If, as the flyleaf anticipates, the book finds its way on to the shelves of research workers in theoretical and solid-state physics, theoretical chemistry and theoretical molecular spectroscopy, one can only hope that they will distinguish clearly between the relatively innocuous mathematics and the appallingly confused physical interpretation which Dr Altmann assigns to it

\section{H. C. Longuet-Higgins}

H. C. Longuet-Higgins is a Royal Society Research Professor at the University of Sussex. and was Professor of Theoretical Chemistry at the University of Cambridge from 1954-67.

gifted high-school students, it would be fairly easy for a Scientific American reader to digest (although any highschool students who can understand it must be extremely select).

The central chapters review much experimental work, especially on plant tumours, which Professor Braun is intimately familiar with, and attempt to produce a unified account of neoplastic growth in plants, amphibians, mammals, and man; from these central chapters I learned a great deal of experimental detail, especially about plant tumours, that was new and interesting. The first and last chapters, however, give the impression of being tacked on later to cover detection, prevention, treatment and molecular biology; they contain some errors, obscurities or restricted perspectives. (Particularly, although some cancers are certainly caused by "chemical substances that have been identified as cancer-producing and which must be identified and removed from the environment", Professor Braun should not say that $70 \%$ or more are thus caused; many, and perhaps most, cancers are caused by certain sexual habits, smoking habits, and gross aspects of diet rather than by contaminants.)

Returning to the central chapters, Professor Braun's main thesis is that tumour cells do not differ importantly from ordinary cells in their nucleotide sequence; all the genetic information can perfectly well remain unchanged, for the key events are, he believes, epigenetic. Tumour cells, he argues, differ from normal cells in the sort of way in which differentiated cell types differ from each other, and he illustrates this argument with examples of plant tumours, frog tumours, mammalian tumours, and certain human tumours.

He describes how teratomas so malignant that a single transplanted cell can cause a tumour may, if implanted into a growing embryo, differentiate into normal organs; how malignant human neuroblastoma cells can be induced to differentiate terminally into RBCs, and how particular viral functions can produce a malignant phenotype without altering the genetic information of the infected cell, and so on. $\mathrm{He}$ describes in great detail the epigenetic changes that enable plant tumours to proliferate, and the ways in in which this neoplastic behaviour can be reversed.

However, there is an alternative way of trying to get order out of the mass of current cancer research; instead of widening the field to be viewed and unified to include all human, mammalian, amphibian and plant tumours, it might be more profitable to narrow it to include only the human carcinomas. Human carcinomas, which comprise $85 \%$ of human cancers, have many characteristics in common with each other, and may differ so fundamentally from other human tumours or from non-human tumours that analogies are misleading. For this reason, I regretted that the discussion of examples included little on human carcinomas, and did not, for example, even mention that gross aneuploidy commonly precedes malignancy.

Finally, there is an over-disparaging review of the potentialities of in vitro mutagenicity assays such as the Ames test, and much sarcastic rhetoric against those who believe that the heritable neoplastic changes in cells are irreversible. The rhetoric is not always convincing, especially as the "somatic mutation" hypothesis which is being attacked is not clearly defined. Thus, this book is not quite the "story of cancer" that I would have liked, but it is still stimulating and interesting. But, as current cancer research contains far too much meaningless detail, the more people who try to stand back and make a story of it the better.

Richard Peto

Richard Peto is at the Department of the Regius Professor of Medicine in the University of Oxford, Radcliffe Infirmary, Oxford, UK. 